EPJ manuscript No.

(will be inserted by the editor)

\title{
Transients in sheared granular matter
}

\author{
Brian Utter ${ }^{a}$ and R. P. Behringer \\ Department of Physics and Center for Nonlinear and Complex Systems, Box 90305, Duke University, Durham, NC 27708 USA
}

October 28, 2018

\begin{abstract}
As dense granular materials are sheared, a shear band and an anisotropic force network form. The approach to steady state behavior depends on the history of the packing and the existing force and contact network. We present experiments on shearing of dense granular matter in a 2D Couette geometry in which we probe the history and evolution of shear bands by measuring particle trajectories and stresses during transients. We find that when shearing is stopped and restarted in the same direction, steady state behavior is immediately reached, in agreement with the typical assumption that the system is quasistatic. Although some relaxation of the force network is observed when shearing is stopped, quasistatic behavior is maintained because the contact network remains essentially unchanged. When the direction of shear is reversed, a transient occurs in which stresses initially decrease, changes in the force network reach further into the bulk, and particles far from the wheel become more mobile. This occurs because the force network is fragile to changes transverse to the force network established under previous shear; particles must rearrange before becoming jammed again, thereby providing resistance to shear in the reversed direction. The strong force network is reestablished after displacing the shearing surface $\approx 3 d$, where $d$ is the mean grain diameter. Steady state velocity profiles are reached after a shear of $\lesssim 30 d$. Particles immediately outside of the shear band move on average less than 1 diameter before becoming jammed again. We also examine particle rotation during this transient and find that mean particle spin decreases during the transient, which is related to the fact that grains are not interlocked as strongly.
\end{abstract}

PACS. 45.70.-n Granular systems - 83.70.Fn Granular solids

\section{Introduction}

Common features of dense, sheared granular materials include the formation of shear bands, which is related to Reynold's dilatancy, and the presence of directionally ori' ented networks of force chains. While the steady state behavior of dense granular shear flows has been the subject of numerous studies, research on transients have typically focused on the effects of cyclic loading and rotation of applied principal stresses in bulk granular samples [4]. Much less is known about the approach to steady state and the effects of changes in imposed shear at the grain scale [1, 2,3.

It is known that forces in compact granular materials are carried preferentially along a network of force chains in which a minority of the grains carry a majority of the load [5]. Imposing shear establishes an oriented network with force chains aligned preferentially so as to resist the applied shear [5] 6. The force network is also correlated with the network of particle contacts, i.e. texture, although one can be highly anisotropic without the other being so [7. Small changes in the contact network are often sufficient to induce substantial changes in the force network; therefore, the force network can be a sensitive

\footnotetext{
a e-mail: utter@phy.duke.edu
}

measure of changes in granular systems. The anisotropy in the force network impacts the trajectories of individual particles during shearing $[\underline{8}$ and creates more mobile and less mobile directions based on shearing history.

Grains are typically confined along certain jammed directions and more free to move along other fragile directions 9. A configuration is jammed if particle rearrangement does not occur under the applied load. Increasing shear stress can often lead to unjamming and flow [10. In addition, a configuration can be unjammed by a small load in a different orientation than the original stress. An analogous situation is a tall stack of blocks which can sustain additional vertical load but easily collapses when pushed from the side. This is referred to as fragility.

When shear is applied to a uniform state, there must be a transient during which the steady state response is achieved. If the imposed shear direction is altered, there must be additional transients during which the force network, texture, and mean particle velocities adjust.

The formation of an oriented network from an initially homogeneous state can be understood by the following argument [7. Small-angle shear deformation through an angle $\phi$ can be decomposed into three parts. Two of these are compression and dilation along axes oriented at $45^{\circ}$ to the direction of shearing. The third is a rotation by $\phi / 2$. Force chains form primarily along the compressional di- 
Brian Utter, R. P. Behringer: Transients in sheared granular matter

rection. In the case of continuous shear deformation, such as that achieved in a Couette apparatus, the heuristic description of the force network also follows from this argument. In this case, the force chains form in response to the initial shear at roughly $45^{\circ}$ to the shear direction, with an orientation that tends to resist further shearing. However, under sufficiently strong shear, the network cannot resist the motion of the shearing surface; it fails as particles roll and slip. Still, on average, the orientation of the strong network remains.

In this paper, we present experimental results on transient behavior in a $2 \mathrm{D}$ Couette shearing experiment. The grains are photoelastic disks which allow measurement of the forces at the grain scale, thus revealing the force chain structures. In these experiments, we study the transients after changing the imposed shear. We generate these transients in one of four ways: 1) by imposing shear on a homogeneous packing; 2) by stopping steady shear and observing the relaxation to a static packing; 3 ) by stopping steady shear, then restarting in the same direction; or 4) by reversing the direction of shear after steady state behavior is reached. This work provides a $2 \mathrm{D}$ analogue to the work by Losert et al. 1]2.

If the system is stopped and shear is re-applied in the same direction, steady state behavior should more or less immediately be produced (assuming slow, quasistatic shearing). That is, aside from some small effect from aging of the force network, the texture should provide a memory of where the system was stopped. If the system is sheared in the opposite direction after establishing a particular texture, a transient should be observed, as the force network is reestablished so as to once again resist shear, after which the particles are again close to a jammed state.

Although one expects force chains to be strong along their primary direction, they are fragile in the transverse direction [11 9] as noted above. The experiments by Losert et al. 1 2, using particle tracking techniques, underscore these expectations through their studies of transients in a 3D Couette flow. In their experiments, restarting shear in the same direction quickly reproduced steady state behavior, while reversing the shear direction created a transient in which particles far from the shearing wheel moved faster than at steady state. They attributed this increased mobility to a softness of granular matter in the direction perpendicular to the principle force axis in agreement with the picture of jammed and fragile directions. Similar results by Calvetti et al.were attributed to an anisotropic contact network induced by the initial shear [3]. Although both of these interpretations are quite reasonable, these experiments could not directly observe changes in the force network. The experiments described here provide imaging and quantitative data for the forces at the grain scale.

\section{Experimental Details}

We show a schematic of the experimental setup in Fig. [1 The granular material (B) is a bidisperse mixture of photoelastic disks (thickness $0.32 \mathrm{~cm}$ ) lying flat on a 2D Plexiglas surface. There are approximately 40,000 grains in a

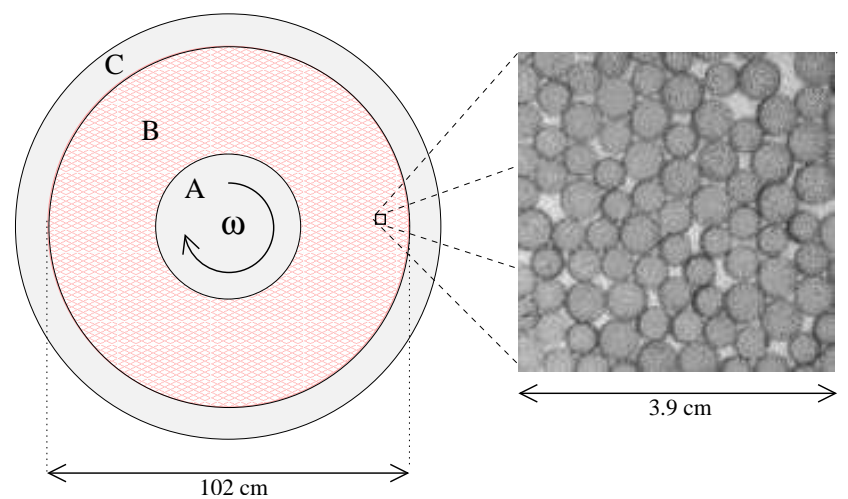

Fig. 1. Top view of the experimental apparatus. The shearing wheel (A) rotates at angular frequency $\omega=2 \pi f$ shearing the granular material (B). The grains are contained by a stationary outer ring (C). On the right, an expanded view of the grains is shown.

ratio of three small (diameter $d_{S}=0.42 \mathrm{~cm}$ ) to one large $\left(d_{L}=0.50 \mathrm{~cm}\right)$ that are contained by an inner shearing wheel (inner radius, $\left.r_{i} \approx 20.5 \mathrm{~cm}\right)(\mathrm{A})$ and a stationary outer ring (outer radius, $\left.r_{o} \approx 51 \mathrm{~cm}\right)(\mathrm{C})$. The shearing wheel is rough at the particle scale and rotates at a frequency $f$ of $0.1-10 \mathrm{mHz}$ or a speed $v \approx 0.013-1.3 \mathrm{~cm} / \mathrm{s}$ at the shearing surface. The data presented here is for $f=1$ $\mathrm{mHz}$ and a mean packing fraction of $\gamma \approx 0.76$ which can be changed between experiments by adjusting the number of particles. We image a subsection of the apparatus ( $\leq$ $10 \%$ ) over long times (see images below). Each particle has a line across its diameter to easily measure its position, orientation, and size. Friction between the grains and the surface on which they lie is decreased by applying a fine powder and has been measured to be two orders of magnitude smaller than the force of a typical force chain 12.

We measure stresses by a "gradient squared method" 12 in which we calculate the mean square gradient $\left\langle G^{2}\right\rangle$ of the image intensity at each pixel and average over the region of interest. This technique is based on the properties of photoelasticity in which increased stress on a particle illuminated between crossed right and left circular polarizers leads to higher density of bright and dark bands and a larger spatial gradient in transmitted light intensity near a contact. We have previously established that $\left\langle G^{2}\right\rangle$ is proportional to the mean force on a particle for the range of forces appropriate to these experiments [12] 7.

The experiment is initially run for multiple revolutions of the shearing wheel to establish a steady state profile. The shearing is then abrubtly stopped. The experiment can then be restarted in the same direction (same shear) or in the opposite direction (reverse shear). This procedure is repeated multiple times to observe average behavior, since fluctuations dominate for individual runs, particularly in the force network. We present measurements of mean particle motion and stresses below. 


\section{Experimental Results}

\subsection{Overview and Organization of Results}

Below, we describe several transient processes. The first of these is when shear is applied to a homogeneous packing. The second occurs when established steady state shearing is stopped. We then consider what happens if shearing is stopped, allowed to relax for a modest length of time, and then restarted in the same direction. Finally, the main focus is determining what happens when steady state shearing is stopped, and then shearing is restarted in the reverse direction.

\subsection{Shearing a Homogeneous Packing}

We first consider the case when the grains are initially uniformly distributed before shearing is applied. In Fig. 2 we show mean displacements of particles at different radial distances from the shearing surface. We bin data in the radial direction and plot mean displacement versus time for each bin. As expected, the disks are quickly pushed outwards to larger values of $r$ as the granular material dilates at the shearing surface and the shear band forms. The tangential displacement also shows a fast response before the shear band forms and particles achieve a slower steady state average velocity. The shear band forms very quickly, within 5-10 s, corresponding to a displacement of under $3 d$ of the shearing surface. After this transient, the evolving force network is qualitatively similar to that seen at steady state.

\subsection{Relaxation}

We now describe the relaxation after steady state shearing is abrubtly stopped. Although it may be expected that relaxation would not occur, that is not the case, as shown below. Other recent results have revealed a long time logarithmic relaxation of the force network [13].

Fig. 3] shows the measured stress as a function of time in three typical cases in which steady state shear was stopped at $\mathrm{t}=0$. In each case, we chose to stop the shearing wheel during a period of large stress. There was clearly a relaxation of stress over a few 10's of seconds. During this time, the overall force network remained largely unchanged, although a few of the stronger force chains broke or relaxed. We show the network relaxation explictly in Fig. 4. Here, the images correspond to when shear is stopped (4A), after $50 \mathrm{~s}$ (4B), and the difference between the first two images $(4 \mathrm{C})$. In Fig. $4 \mathrm{C}$, white indicates an increase of force, and dark indicates a decrease of force. In addition, the bars on some of the particles close to the shearing surface shifted slightly. The relaxation was confined to the shear band with small displacements observed in the grains within the first few layers of particles. Note that there was very little motion outside the first few layers and, even close to the shearing surface, measureable changes in the contact network were small.
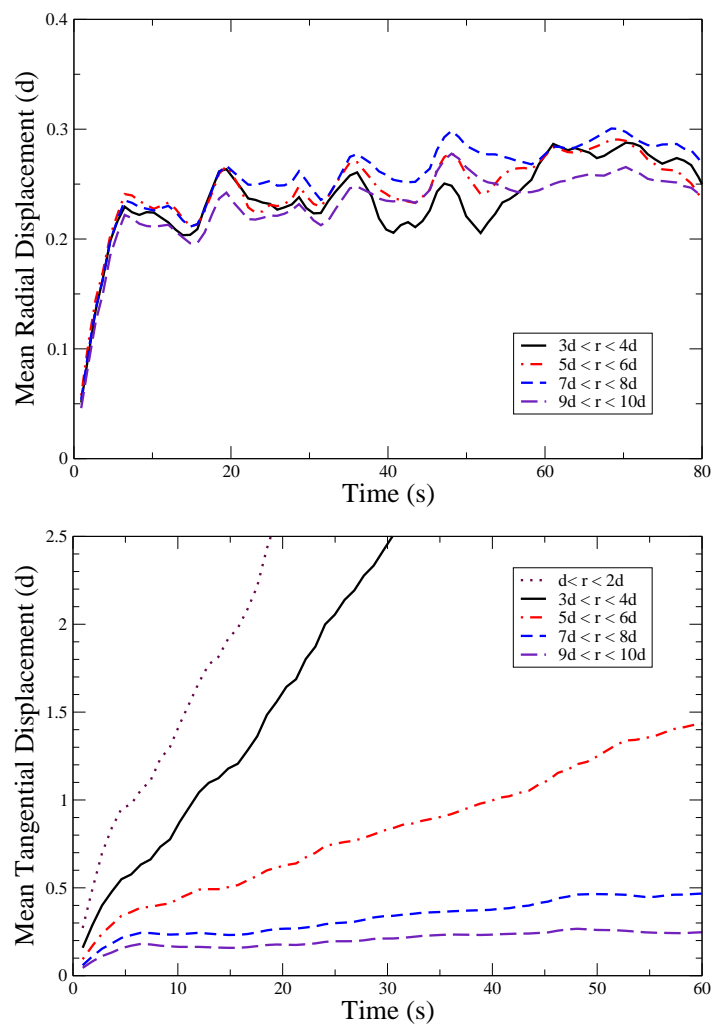

Fig. 2. Mean radial and tangential displacements during a transient starting from a uniform spatial distribution of particles $(f=1 \mathrm{mHz})$. After a transient of $5-10 \mathrm{~s}$ (shearing surface dis '

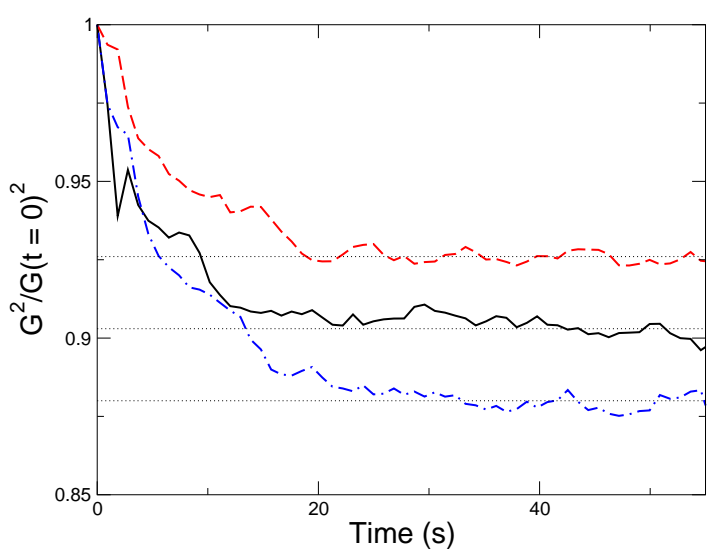

Fig. 3. Decay in mean stress vs. time. Stress is measured using a "gradient squared method" as described in the text. In three instances, we stopped the shearing $(\mathrm{t}=0)$ at a point of large stress within the field of view, and we then observed the relaxation of stress. 

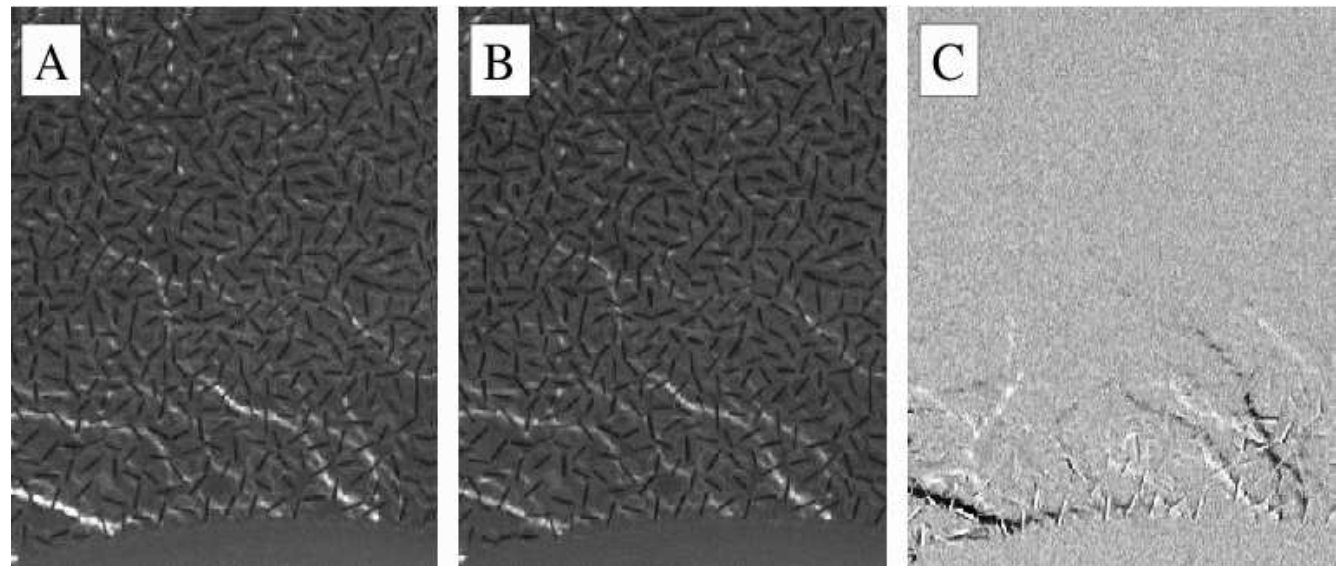

Fig. 4. Images shown (A) immediately after stopping steady shear to the left and (B) 50 seconds later. (C) The difference between the center and left images shows that particle motion is small and limited to grains close to the shearing wheel. Stronger force chains have weakened (black) displacing the force to other chains (white).

\subsection{Restarting Shear in the Same or Reverse Direction}

In this section, we study the effect of restarting shear either in the same or opposite direction as a previously applied shear. In particular, we ask what transients occur before reaching steady state. We show that restarting shear in the same direction leads to nearly immediate recovery of steady state behavior. Restarting shear in the opposite direction leads to a transient of approxiamtely 50-100 s (15-30 $d$ displacement of shearing surface) during which particles outside of the shear band are more mobile and observed stresses reach further into the bulk.

When shearing is reversed, the anisotropic force network must readjust. Fig. [5] shows typical force chains during steady state shearing along an initial direction (55) and the force network with the shear reversed (5B). The white arrow represents the shear direction and the straight lines indicate the approximate orientation of the large force chains. As described above, these form at approximately $45^{\circ}$ to the direction of shear, so as to resist the shear force. The fragile directions are then at $90^{\circ}$ to these lines 119. Note that upon reversing shear, the strong force direction switches to approximately align with the previous fragile direction. For comparision, Fig. [5C shows an image of the particles at the same magnification as that of the photoelastic force images.

We show velocity profiles immediately after restarting with the same or reverse shear in Fig. [6] The mean tangential velocity $v_{\theta}$ is plotted versus distance from the shearing wheel in units of the mean particle scale $d=\left(d_{S}+d_{L}\right) / 2$ 14. Quantities are measured as functions of the radial distance $r$ (where $r=0$ corresponds to the shearing surface) and averaged over azimuthal angle, $\theta$. Data is collected for the first 28 seconds after restarting shear, corresponding to a displacement of $\approx 10 d$ of the shearing surface. The curve for steady state behavior corresponds to a longer data run (1000 s), well after the initial transient has occurred. The velocity profile when restarting shear in the same direction is nearly indistinguishable from steady state behavior. This is expected for quasistatic flows. Although we observe
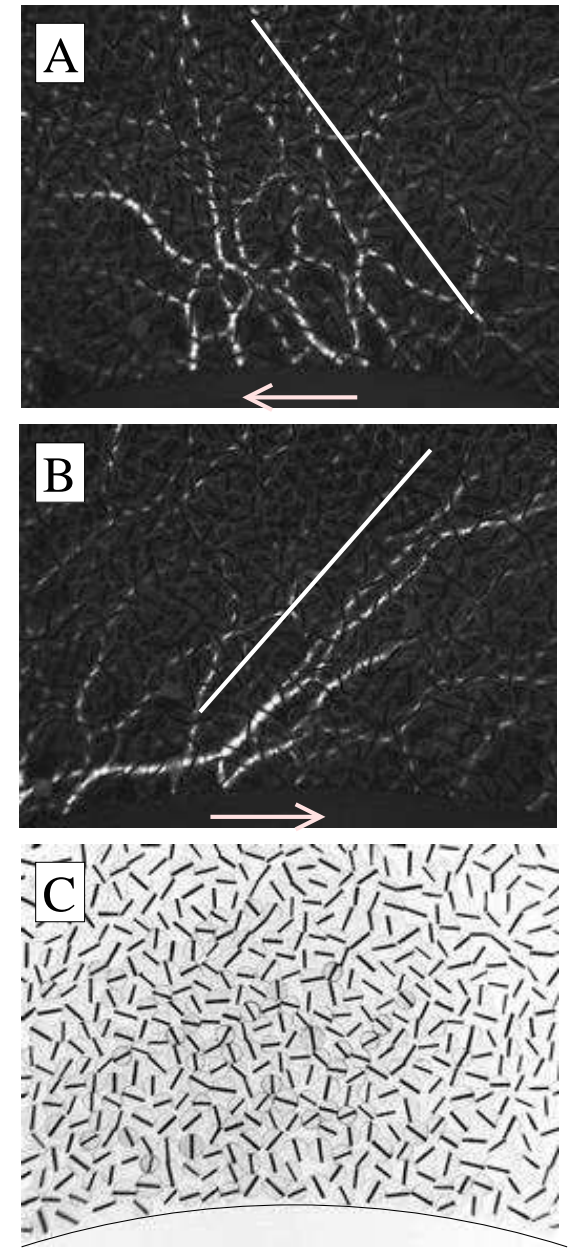

Fig. 5. Stress images during (A) same shear and (B) reverse shear. The arrow indicates the shearing direction and the solid lines indicate the approximate orientation of the strong force network. (C) An image showing the grains at the same magnification. 

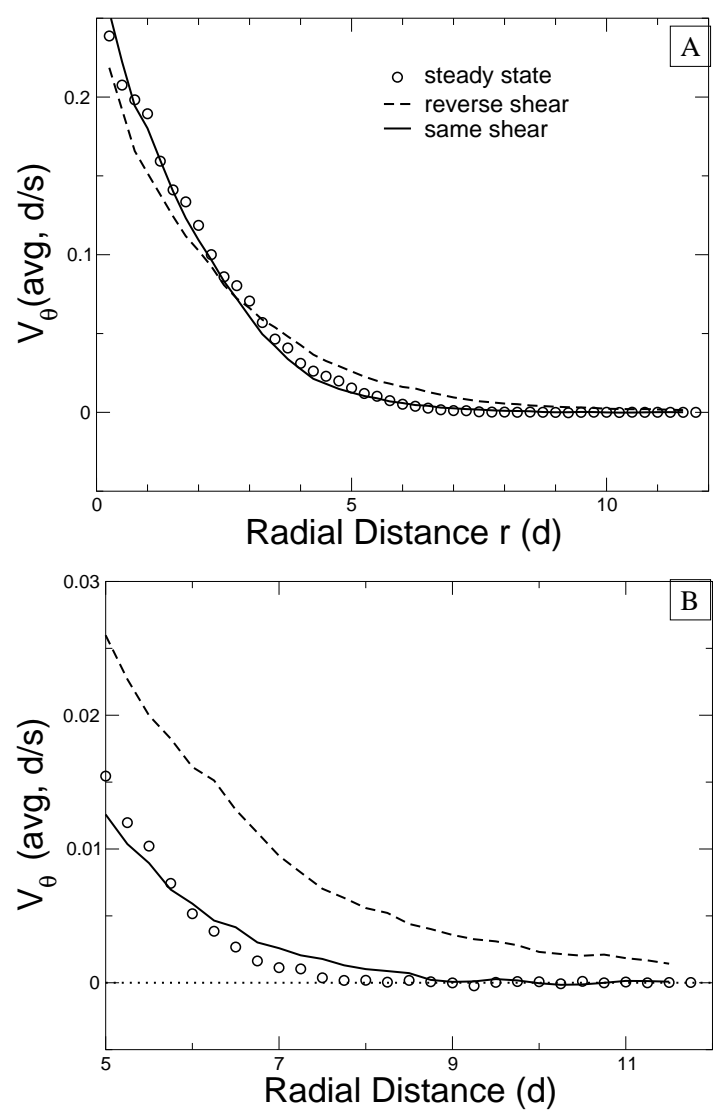

Fig. 6. Mean tangential velocity vs. radial distance from the shearing wheel for six runs $(f=1 \mathrm{mHz})$. Continuing the same shear direction (solid line) is indistinguishable from the steady state profile (o) while reversing shear (dashed line) is clearly different. The figure on the right is an expanded view of that on the left. Particles that were jammed at steady state (far from shearing wheel) are now mobile, i.e. granular matter is fragile when forces are applied in a direction that is normal to the established texture. Here, the shearing rate is $f=1$ $\mathrm{mHz}$. These data were obtained in both cases during the first 28 seconds after (re)imposing shear.

some weak relaxation of the stress network (Fig. 3) substantial relaxation of the contact network does not occur. Hence, the initial velocity profiles and the profiles after restarting in the forward direction are virtually identical. That is, the force network experiences some aging once shearing is stopped [13, but there is essentially no change in the contact network, so the system can be regarded as quasi-static under shearing.

When shearing is initiated in the reverse direction, there is a transient in the velocity profile. Grains outside of the original shear band become unjammed and, consequently, move more because they are now being pushed along the fragile direction. The effect is reversed for grains near the shearing surface. These grains are initially pushed away from the shearing surface because there is initially no strong force network to resist this type of motion. Consequently, they are not pulled along as strongly as they would be in an established shear band. Fig. $6 \mathrm{~B}$ shows an expanded view of Fig. 6A. There, it is clear that particles which were previously jammed $(7 d<r<12.5 d)$ are now mobile.

We also examine where significant changes in the force network occur, following the procedure used in Fig. 4C. In Fig. 17] we show difference images between the state immediately after steady state shearing was stopped and the state $28 \mathrm{~s}$ after restarting shear $(10 \mathrm{~d}$ displacement of shearing surface). These images show changes in the force network during the transient and contrast the two cases of shearing in the same and reverse directions. For each run, we use the magnitude of the difference image (i.e. deviation from grey in Fig. $4 \mathrm{C}$ ) such that chains that form or disappear in the intervening time both contribute equally to the resulting intensity. Thus, in Fig. 7 white indicates a change in the force network and black indicates where no change occured. In both difference images (Fig. [7A and B), we show the average from 6 runs and adjust the contrast to highlight the changes at the edge of the shear band. We also show a scale bar of length $10 d$. In each case, significant changes in the force network are predominantly confined to the shear band $(r<6 d)$ as expected. However, upon reversing shear, changes in the force network reach further into the bulk, including the region where particles are initially more mobile $(6-12 d)$. In Fig. $7 \mathrm{C}$, we plot the (unadjusted) mean intensity versus radial distance from the shearing surface for the data used to construct Fig. 77A and $\mathrm{B}$. There it is evident that changes in the force network reach further into the bulk when shear is reversed.

We now ask how long the increased mobility state lasts, and how far particles move before becoming jammed again. In Fig. 8, we show the mean displacement versus time, averaged over four runs, for particles at different distances from the shearing surface when the shearing direction is reversed. After an initial transient $(\mathrm{t} \lesssim 60$ $\mathrm{s})$, trajectories follow $r \Delta \theta=v_{a v g} \Delta t$. The straight lines are fits to the displacement versus time data after the transient (100-325 s). The short line segments at the end of each curve indicate the expected slope $v_{\text {avg }}$ based on the steady state velocity profile in Fig. 6] Deviations between the slope of the short line segments and the straight lines may indicate that additional rearrangement occurs after $t=325 \mathrm{~s}$. We also note that we compare short transient data runs to a separate steady state run, and slight variations in packing structure and velocity profiles are possible. However, we focus on the most pronounced transient which occurs within the first 50-100 s. Particles that were previously jammed or moving slowly initially move relatively fast before reaching steady state conditions. This additional displacement due to the increased mobility during the transient ( $y$ intercept of straight line fits) is small, with displacements of less than $1 d$ needed to become jammed again. The existence of the additional displacement is qualitatively the same as that found by Losert et al. 1,2 , but the additional displacements are significantly smaller in our experiment. The timescale for readjustment is 50-100 seconds, corresponding to a displacement of the shearing wheel of $15-30 d$. Note that the transient for reversal is significantly longer than when ap- 

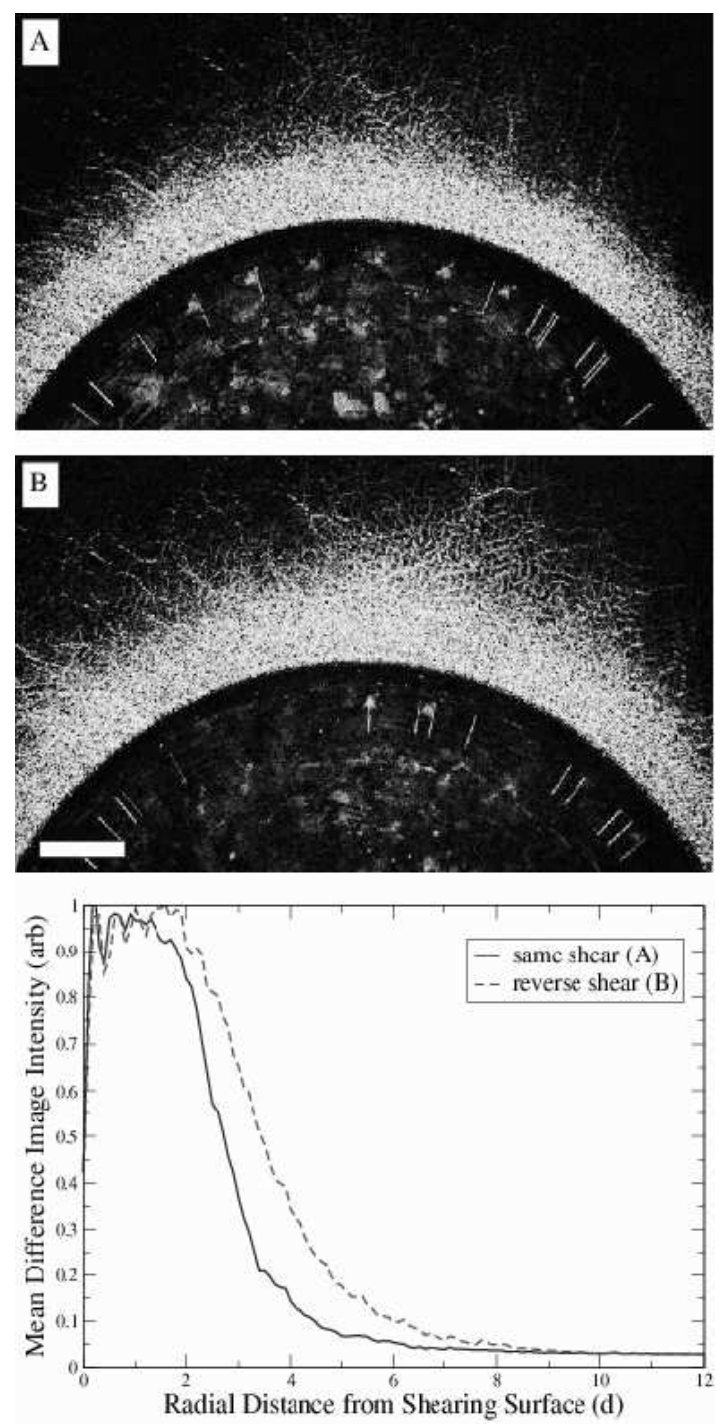

Fig. 7. Difference images showing the magnitude of the changes in force network during transients for (A) same shear and (B) reverse shear. Each image is an average of six runs. The contrast has been enhanced to emphasize the differences at the edge of the shear band. In (C) we show the mean intensity of the original images versus radial distance from the shearing surface.

plying shear to a homogeneous packing (Fig. 2). In agreement with Fig. 6] particles close to the wheel, initially move slower after shear reversal than at steady state, although the lag in displacement is small compared to the fluctuations in the steady state.

In Fig. 9, we have taken the data in Fig. 8 and removed the long time behavior (straight lines). Thus, the data in Fig. 9 eventually fluctuate around 0 and represent deviations from the mean steady state over time. At early times, negative values of the displacement show where particles have lagged and positive values show where they have advanced further than expected based on extrapolating back from the long-term steady state. After the 50-100 s transient, not only are steady state velocities reached, but also

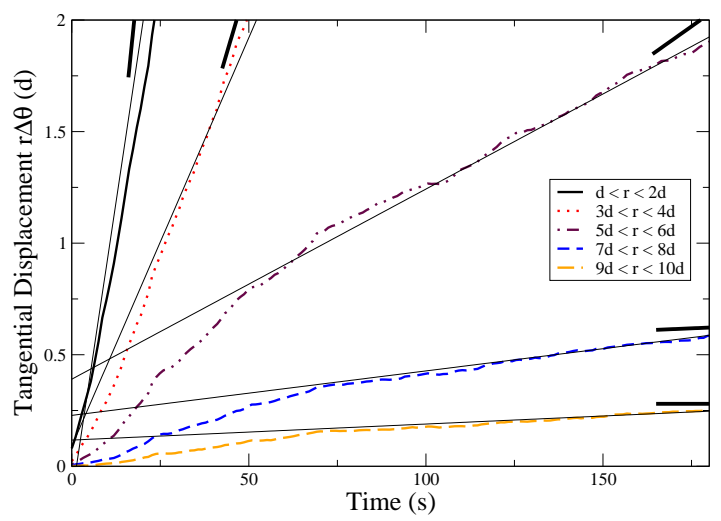

Fig. 8. Mean displacement versus time for particles at different radial distance after reversing shear. The data is for an average of four runs. Particles far from the shearing wheel $(r \geq 7 d)$ move a fraction of a particle diameter $d$ before reaching steady state velocity or becoming jammed again. The short line segments indicate the steady state slope expected from Fig. 6]

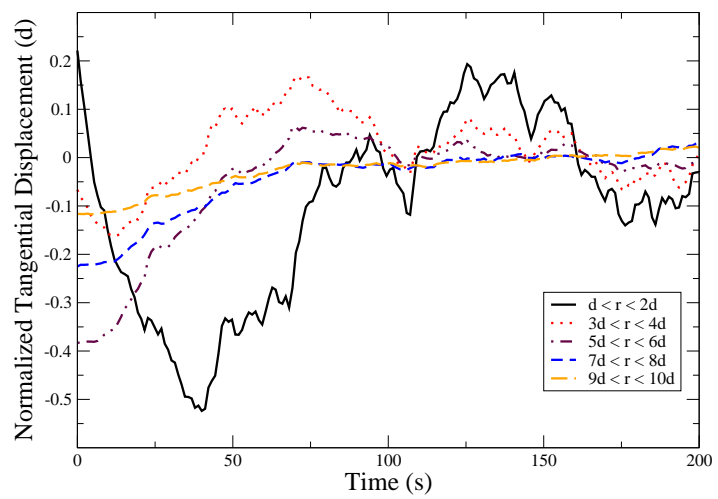

Fig. 9. Mean tangential displacement with the steady state fits (straight lines in Fig. 8) subtracted off. At long times, the curves fluctuate around zero.

the mean motion of particles at different distances from the shearing is coordinated and grains at different $r$ advance or lag in unison.

We also measure the mean stress during these transients using the $\left\langle G^{2}\right\rangle$ technique described above. Fig. 10] shows the mean stress integrated over all particles in $r<$ $10 d$, for an average of six runs. There it is clear that for $\mathrm{t}<7 \mathrm{~s}$ there is no observable stress for the case where the shear direction has been reversed. Although the fluctuations are different for each run, the initial quiescent period is observed in each case and for multiple packing fractions $(\gamma=0.755,0.76,0.765)$. This is similar to the $3 \mathrm{D}$ results of Toiya et al. [2], despite the fact that in 3D, there is a compaction of the system [1]. Note that the stress readjusts on a smaller time scale than that for velocity relaxation. Qualitatively, the force network appears similar to the steady state network after $t \simeq 10 \mathrm{~s}$. Additional small adjustments of the force network might occur dur- 


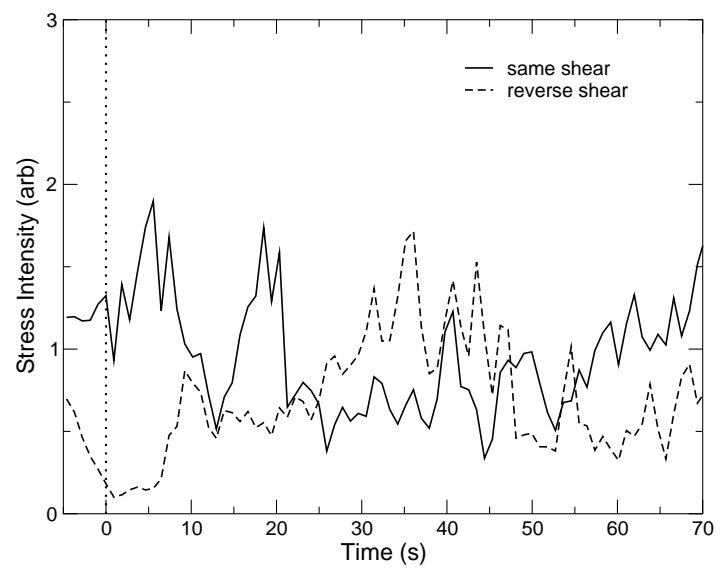

Fig. 10. Mean stress vs. time within the shear band $(r<10 d)$. There is initially no measurable stress upon reversing shear $(t<7 s)$. Data shown is an average of six runs.

ing the velocity relaxation, but they are not evident due to the strong fluctuations.

We study particle rotations during the transients in Fig. 11A, where we show the mean particle rotation rate versus distance from the shearing wheel. The negative values close to the shearing wheel reflect particles in contact with the wheel which are counter-rotating (Fig. 11B). If all particles in the innermost layer were stationary and counter-rotating with the shearing wheel, the mean rotation rate or spin, $S$, would be $\approx-0.6 \mathrm{rad} / \mathrm{s}$, or in the scaled form $S 2 \pi f r_{i} / d=-1$. The positive peak for $r \approx 1$ is due to the counter-rotation of the second layer (Fig. 11B) [6]. Further into the bulk $(r>d)$, the mean rotation is clockwise, which might be expected for frictional particles in a shear flow (Fig. 11C). That is, for the intermittent motion of a grain in the bulk, it is most likely that the neighbor further from the shearing surface is nearly stationary while the neighbor closer to the shearing surface moves to the left, leading to clockwise rotation of the grain. The data is qualitatively similar when restarting shear in the same or reverse directions, but the magnitudes of the peaks is somewhat smaller during reverse shear since they are not interlocked as strongly. In Fig. 11D, we show the difference in mean spin vs. $r$ (same shear - reverse shear) for five individual runs. Although the data is noisy, there is a clear trend of positive values at $r<0.5$ and negative values at $r \approx 1$ indicating the decrease in the magnitude of the particle spin under reverse shear seen in Fig. 11A.

\section{Conclusions}

We have studied the behavior of transients in a 2D granular Couette shear experiment. We find that when we restart shear in the same direction as previous steady state shearing, no significant transients occur. Even though some slow relaxation of the force network occurs when the system is stopped, there is a minimal transient on restarting
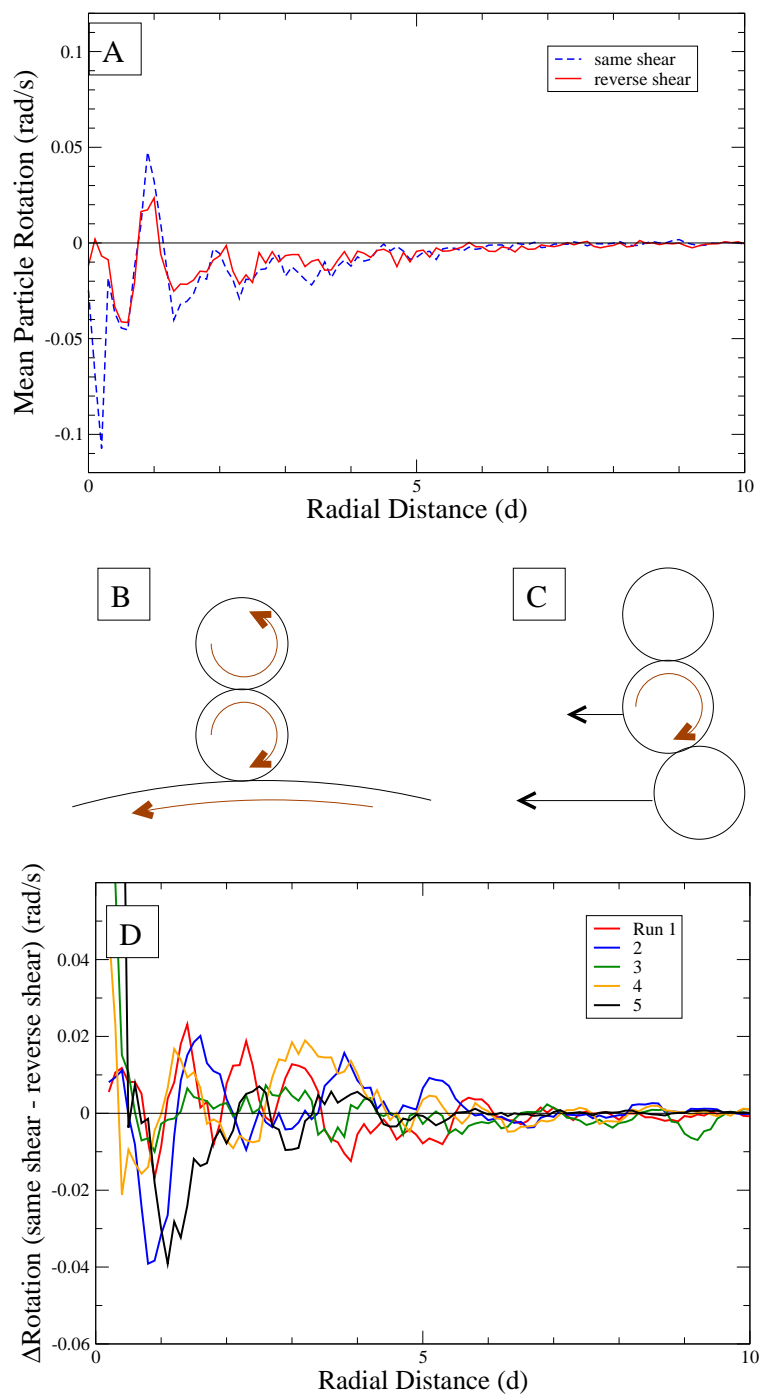

Fig. 11. (A) Mean particle rotation versus radial distance. The mean behavior is influenced by (B) particles counterrotating close to the shearing wheel and $(\mathrm{C})$ particles rotating clockwise due to shear $(r>1)$. In (D), we show the difference $\Delta$ Rotation which is the data for the reverse shear rotation subtracted from the same shear rotation for five individual runs.

because there are no significant changes in grain positions. In this sense, the process is quasistatic. When we reverse the shearing direction, transients do occur as the force network reforms. During this process, grains outside of the shear band are more mobile since they are pushed along an initially fragile direction. Photoelastic stress images support this picture. Mean grain displacements of less than a particle diameter are sufficient for particles far from the shearing surface to become jammed again and reach a nearly steady state stress structure. Immediately after reversal, there is no measurable stress, since there is no pre-existing force network to resist shearing. During the transient however, changes in the force network for reverse shear reach further into the bulk as compared to what occurs for restarting shear in the same direction. 
The strong force network is reestablished after displacing the shearing surface by $\sim 3 d$. Steady state velocity profiles are reached after a displacement of $\leq 30 d$. Reversing the shear decreases the magnitudes of the particle spins slightly since grains are not interlocked as strongly, but the spin profile still maintains the same qualitative shape, with an overall reversal of sign.

Acknowledgement We appreciate a number of helpful discussions with Prof. Wolfgang Losert. This work has been supported by the National Science Foundation through grants DMR-0137119, DMS-0204677 and DMS-0244492, and by NASA grant NAG3-2372.

\section{References}

1. W. Losert and G. Kwon, Advances in Complex Systems 4, 369 (2001).

2. M. Toiya, J. Stambaugh, and W. Losert, unpublished (2003).

3. F. Calvetti, G. Combe, and J. Lanier, Mechanics of Cohesive-Frictional Materials 2, 121 (1997).

4. See, for example, J. R. F. Arthur, T. Dunstan, A. Dalili, and R. K. S. Wong, Powder Technology 6589 (1991) and L. Rothenburg, R. J. Bathurst, and A. A. Berlin, Powders and Grains 93, ed. C Thornton, 147 (1993).

5. D. Howell, R. Behringer, and C. Veje, Chaos 9, 559 (1999).

6. C. Veje, D. Howell, and R. Behringer, Physical Review E 59, 739 (1999).

7. J. Geng, G. Reydellet, E. Clement, and R. P. Behringer, Physica D 182, 274 (2003).

8. B. Utter and R. P. Behringer, Physical Review E 69, 031308 (2004).

9. M. Cates, J. Wittmer, J.-P. Bouchaud, and P. Claudin, Physical Review Letters 81, 1841 (1998).

10. A. J. Liu and S. Nagel, Nature 396, 21 (1998).

11. F. Radjai, D. Wolf, M. Jean, and J.-j. Moreau, Physical Review Letters 80, 61 (1998).

12. D. Howell, R. Behringer, and C. Veje, Physical Review Letters 82, 5241 (1999).

13. R. R. Hartley and R. P. Behringer, Nature 421, 928 (2003).

14. We use the mean of the two disk diameters $\left(d_{S}+d_{L}\right) / 2$ as the relevant length scale. Also acceptable would be to use the mean particle size, i.e. $\left(3 d_{S}+d_{L}\right) / 4$ since our medium is a mixture of small and large particles in a ratio of $3: 1$. In our experiment, this would decrease the value of $d$ and rescale $v$ and $r$ by $4.3 \%$. 